\title{
Reversible and irreversible polarization processes in ferroelectric ceramics and thin films
}

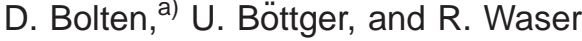 \\ Institut für Werkstoffe der Elektrotechnik, University of Technology RWTH Aachen, \\ D-52056 Aachen, Germany
}

(Received 18 June 2002; accepted 13 November 2002)

\begin{abstract}
In this article, the separation between reversible and irreversible polarization where the reversible polarization component is determined by capacitance-voltage curve measurements, is used to characterize ferroelectric materials. After giving a thorough foundation of the method, it is used to investigate the influence of the composition on the reversible and irreversible polarization contributions in ferroelectric thin films and/or bulk ceramics. The reversible polarization is also monitored during fatigue. A comparison to bulk ceramics suggests that the domain wall motion in ferroelectric thin films is reduced compared to bulk ceramics. (c) 2003 American Institute of
\end{abstract} Physics. [DOI: 10.1063/1.1535748]

\section{INTRODUCTION}

When an oscillating electric field of sufficient strength (i.e., $E \gg E_{c}, E_{c}$ coercive field) is applied to a ferroelectric material, the accompanying changes in the electric polarization are hysteretic. The hysteresis appears due to a complicated combination of reversible and irreversible polarization processes in the material as a result of the applied field.

There are basically two mechanisms that lead to irreversible changes in the ferroelectric polarization. First, a lattice cell can switch from one thermodynamically stable configuration (say $\left.+P_{\text {sat }}\right)$ to another $\left(-P_{\text {sat }}\right)$. This process is inherently not reversible unless some critical field is exceeded. The second mechanism is irreversible domain wall motion, elucidated by a simple model based on the assumption that the domain wall is moving through some kind of potential generated by the interaction of the domain wall with randomly distributed defects of the lattice, e.g. dislocations, dopant ions, vacancies, etc. ${ }^{1,2}$ The possibility of such interactions is evident considering that a domain wall is necessarily associated with a local strain field. A purely elastic interaction with defects that produce strain fields (dopant ions, dislocations, vacancies) is therefore plausible. Boser et al. analyzed the interaction between a dopant ion that is treated as an elastic dipole with the stress field surrounding a $180^{\circ}$ domain wall in analogy to similar calculations for ferromagnetic Bloch walls. ${ }^{3}$ Even though the calculated interaction force between a single dopant ion and a domain wall turns out to be relatively small, the overall force could be quite substantial due to the large number of interacting dopant ions in a doped ferroelectric. Another possibility is the interaction of charged domain walls (with div $P \neq 0$ ) with charged defects.

Reversible domain wall motions result when the domain wall is moving inside a local minimum of the random potential. The domain wall contributes irreversibly when the force exerted on the wall by the external field is big enough to drive the domain wall over a local maximum of the potential,

${ }^{a)}$ Electronic mail: bolten@iwe.rwth-aachen.de thus preventing the wall from returning to its initial position when the external force is removed.

There are three approaches to experimentally obtain information about reversible and irreversible processes: the measurements of Rayleigh loops, the measurement of "recoil curves" (to be explained shortly), and the measurement of the small signal capacitance.

The Rayleigh law, i.e., the observation that subcoercive hysteresis loops can be described by a relatively simple set of equations, allows a simple discrimination between the reversible and irreversible polarization contributions by virtue of the initial permittivity $\varepsilon_{\text {init }}$ and the Rayleigh constant $\alpha$ which describe the functional form of a Rayleigh loop. For ferromagnetic materials, a wealth of theories exist that try to relate microstructural features to the Rayleigh constant and the initial permittivity that can serve as a guideline for ferroelectric materials as well. Damjanovic et al. showed that the Rayleigh law can also be used to describe the subcoercive hysteresis loop in ferroelectric and piezoelectric materials. ${ }^{1,2}$ Boser $^{4}$ established an inverse proportionality between the Rayleigh constant $\alpha$ and the doping concentration $N$, that holds for iron doped barium titanate ceramics. The same relation appears to be valid for donor doped PZT thin films. ${ }^{5,6}$

A direct method to measure reversible and irreversible contributions most appealing to intuition is the measurement of subloops and termed "recoil curves" in the following. To measure the irreversible polarization, the sample is first driven to a state $(E, P)$ on the hysteresis curve. The irreversible polarization $P_{\text {irr }}$ is then defined as the polarization remaining when the external field is changed back to zero along a recoil curve. The reversible polarization, $P_{\text {rev }}$, is calculated as the difference between $P$ and $P_{\text {irrev }}$. This is only rigorously true if no irreversible changes occur along the recoil curve. An analogous procedure is used in the field of ferromagnetics to characterize the reversible and irreversible magnetization. ${ }^{7}$ A more detailed description of the measurement procedure will be given shortly.

With the last method, the small signal capacitance is measured by superimposing an ac electric field with a small 
amplitude ( $\approx 1 \mathrm{kHz}, 50-100 \mathrm{mV}$ ) over a (slowly varying) dc field that traces the hysteresis loop. The capacitance is then determined by the measurement of the component of the current that is phase shifted by $90^{\circ}$ with respect to the driving ac voltage. Indeed, the effective slope of a local subcycle is determined in this way. As a result one obtains a so-called capacitance-voltage dependence $(C-V)$. In the limit of vanishing amplitude of the applied ac signal, one can expect that the domain walls move only reversibly inside a local minimum of the above mentioned interaction potential, so that the measured response is a measure for the reversible contribution of the domain walls at a given dc bias (and of the domain configuration at that given dc bias). Since ideally no irreversible processes occur during the measurement of a minor loop (in the limit $E_{\mathrm{ac}} \rightarrow 0$ ), the measurements are made under the condition of constant irreversible polarization $P_{\text {irrev }}$. This approach is very similar to the measurement of the reversible susceptibility used in the study of ferromagnetic materials where the incremental susceptibility $\Delta M / \Delta H$ (i.e., the slope of a minor loop) defines, in the limit of $\Delta H$ $\rightarrow 0$, the reversible susceptibility $\chi_{\mathrm{rev}}{ }^{8}$ Since by definition $C=d Q / d U$, a polarization can be extracted from a $C-V$ curve by:

$$
P_{\mathrm{rev}}=\frac{1}{A} \int C(V) d V,
$$

where $A$ denotes the area of the sample. $P_{\text {rev }}$ can be interpreted as the reversible polarization contributions along the hysteresis curve, again in complete analogy to the magnetic case. $^{9}$

In this article, the latter method is compared to results obtained with the recoil method. It will be shown that both methods generate almost identical results. The integrated $C-V$ curve method is then applied to study the dependence of the reversible and irreversible polarization contributions on the composition. Their change during a fatigue treatment will also be analyzed. A comparison with the response of bulk ceramics will provide evidence for reduced domain wall contributions in ferroelectric thin films.

\section{EXPERIMENT}

The PZT ceramics investigated in this work were obtained from the University of Karlsruhe, Germany. More details on the samples can be found in Ref. 10. The samples were prepared using the mixed-oxide process. The ceramics were sintered at $1250^{\circ} \mathrm{C}$. Soft PZT ceramics with a neodymium dopant concentration of $2 \% \mathrm{~Pb}_{0.98} \mathrm{Nd}_{0.02}\left(\mathrm{Zr}_{x} \mathrm{Ti}_{1-x}\right) \mathrm{O}_{3}$ were prepared. The zirconium content $x$ was varied from $x$ $=0.48$ (tetragonal) to $x=0.6$ (rhombohedral). The grain size of the ceramics varied between 3 and $4 \mu \mathrm{m}$ and the relative density was always above $96 \%$ of the theoretical density.

The preparation of the $\mathrm{PbZr}_{x} \mathrm{Ti}_{1-x} \mathrm{O}_{3}$ films of various $\mathrm{Zr} / \mathrm{Ti}$ ratios is described in detail in Ref. 5. All thin films exhibited good phase purity. No second phases could be detected with $\mathrm{x}$-ray diffraction analysis.

Most hysteresis curves presented in this work were measured with the aixACCT TF analyzer 2000, a computer based measurement tool to characterize ferroelectric thin films. The system allowed hysteresis measurements in the dynamical range between $1 \mathrm{~Hz}$ and $1 \mathrm{kHz}$ with a maximal amplitude of $10 \mathrm{~V}$.

In the same manner, ferroelectric bulk samples were characterized. In this case, however, a special high voltage probe head was used to protect the electronic circuits in the case of a dielectric breakdown of the sample. The amplitude and frequency range was then determined by the high voltage amplifier used (Trek Model 609D-6) whose input voltage was provided by the TF analyzer. With the Trek 609D-6, a maximal voltage of $4 \mathrm{kV}$ could be applied to the sample. Due to the maximal current of $20 \mathrm{~mA}$ that this type of amplifier could drive, the upper limit of the dynamic range was just a few Hertz.

For the measurement of recoil curves, the excitation signal was generated by an arbitrary waveform generator (amplified with a high voltage amplifier in the case of bulk ceramics). The current response of the sample was amplified with a Keithley Model 428 current amplifier whose output was recorded with a digital oscilloscope (Tektronix, TDS 684C). The recorded data could be transferred to a computer via the IEEE488 bus for further procession.

The capacitance-voltage dependence was measured with an HP 4284A LCR bridge. The equipment used allowed the variation of the amplitude $U_{\text {ac }}$ of the small-signal measuring signal between $5 \mathrm{mV}$ up to $10 \mathrm{~V}$. By using the bias feature of the bridge, a dc voltage could be superimposed on the small measuring signal, thus allowing to measure the permittivity at different polarization states (which correspond to different points of the ferroelectric hysteresis). By changing the dc bias in a step-like fashion, a complete $C-V$ curve was measured. Since this measurement was controlled via the IEEE488 bus with a computer, the dc bias could only be changed slowly, resulting in a large-signal frequency of $\sim 100 \mathrm{mHz}$.

For higher frequencies of the large signal, a different method had to be used. Here, the slowly varying large signal was generated with an arbitrary waveform generator (Wavetek 395). On this signal, a small-signal measuring voltage (the reference channel of a PerkinElmer DSP Model 7280 lock-in amplifier) was superimposed electronically and then fed to the sample. The answer of the sample was then applied to the input of the lock-in amplifier. With this setup a dynamic range of the large signal excitation between a few $\mathrm{mHz}$ up to $10 \mathrm{~Hz}$ was possible.

\section{RESULTS}

\section{A. Preliminary remarks}

The concept that $C-V$ curves can be used to determine the reversible polarization is based on the notion that small external fields will only result in small reversible displacements of the domain walls inside the material, and that by reducing the amplitude of the external field existing irreversible processes can be suppressed completely. The reversible capacitance $C_{\text {rev }}$ can therefore be defined as:

$$
C_{\mathrm{rev}}(E):=\lim _{E_{\mathrm{ac}} \rightarrow 0} C\left(E, E_{\mathrm{ac}}\right),
$$



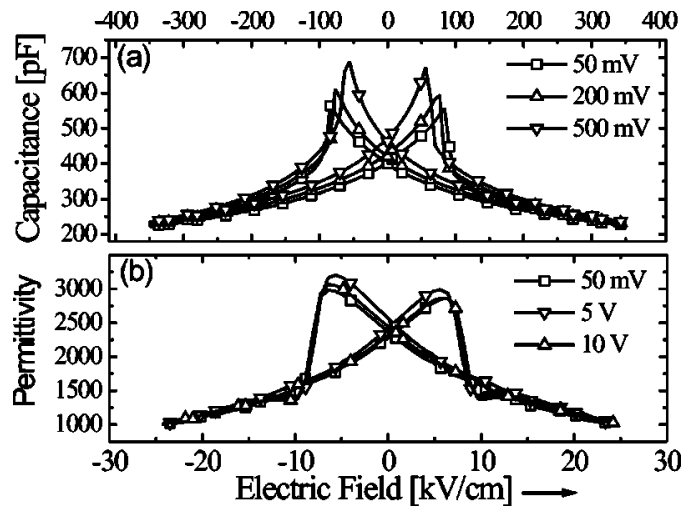

FIG. 1. Small-signal amplitude dependence of the capacitance-voltage curve of a PZT 30/70 film (150 $\mu \mathrm{m}^{2}$ electrode size, $200 \mathrm{~nm}$ thickness) (a) and a PZT bulk ceramic of morphotropic composition (b) (thickness 250 $\mu \mathrm{m})$.

where $E_{\text {ac }}$ denotes the amplitude of the oscillating small signal excitation used to determine the capacitance and $E$ the current external bias. This is the most precise definition but not the best feasible for experimental measurements. Ideally, the reversible contributions should be measured at vanishing external field strengths. However, the measurement equipment used requires a nonzero excitation. According to the definition given above, a complete measurement of a $C-V$ curve would require the measurement of the ac amplitude dependence of the capacitance at all bias fields and their subsequent extrapolation to $E_{\mathrm{ac}}=0$. This measurement procedure, however, could have a significant impact on the measured properties of the material due to the long exposure to $\mathrm{dc}$ voltage stress. Therefore, the question needs to be addressed if the reversible contributions can be approximated by measurements with a small but nonvanishing signal that is still big enough to allow the determination of the small signal capacitance with sufficient precision.

Figure 1 displays the amplitude dependence of the capacitance for a (a) PZT 30/70 film (200 nm thickness) and (b) a PZT bulk ceramics of morphotropic composition (250 $\mu \mathrm{m}$ thickness). For low amplitudes of the superimposed ac signal the amplitude dependence of the capacitance was only very small for both the thin film and the bulk ceramic sample. The lowest amplitude compatible with sufficient measurement precision (determined by the LCR bridge used) could therefore be taken as a good approximation to the exact value of Eq. (2).

Integrating the measured capacitance-voltage curve gives by virtue of Eq. (1) the reversible polarization. Figure 2 displays a typical result (of a PZT 30/70 film). In contrast to a normal hysteresis curve, the integrated $C-V$ curve (labeled with $P_{\mathrm{rev}}, f_{\mathrm{ac}}=100 \mathrm{kHz}, U_{\mathrm{ac}}^{\mathrm{rms}}=25 \mathrm{mV}$ ) was very narrow and the polarization values reached are also much smaller. The reader might wonder why the reversible polarization contributions exhibited a hysteresis, which is counterintuitive to the very notion of a reversible process. One should keep in mind that during the measurement of a $C-V$ curve, a slowly varying dc bias was superimposed over the measuring signal, i.e., the normal ferroelectric hysteresis was traced out entailing irreversible changes in the domain con-

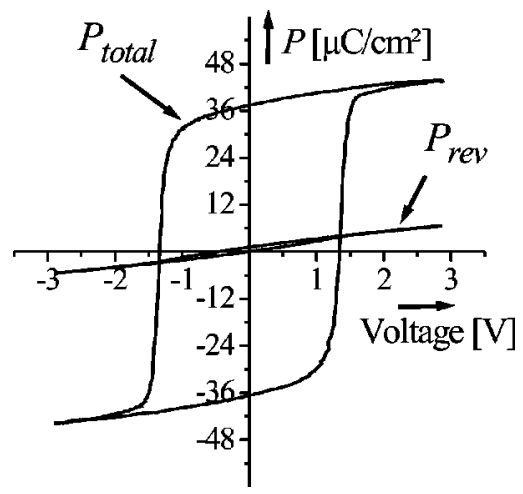

FIG. 2. Comparison of reversible to total polarization for the same film. The reversible polarization was obtained by integrating a $C-V$ curve of a PZT 30/70 film.

figuration. Thus, the integrated $C-V$ curve, i.e., the reversible polarization contribution, has to be interpreted pointwise, being characteristic for the electric field and the corresponding domain configuration at that point, thereby rendering a hysteretic overall appearance perfectly acceptable.

Figure 2 also shows a comparison between the reversible polarization obtained from an integrated $C-V$ curve and a dynamically recorded hysteresis. The hysteresis was traced in both cases with a sine wave with $f_{\text {hys }}=10 \mathrm{~Hz}$. Notice that in the saturation regime the reversible polarization had the same slope as the dynamic hysteresis. This can be taken as strong evidence that the integrated $C-V$ curve is indeed a measure for reversible polarization processes since according to common understanding in the saturation regime only reversible polarization processes occur (mainly due to ionic and electronic polarization processes).

Thus, the integrated $C-V$ curve can be interpreted as a measure for reversible polarization processes inside the ferroelectric material. This interpretation, however, is only valid if the capacitance is measured utilizing sufficiently small oscillation amplitudes of the super-imposed ac signal. Since both the small-signal capacitance and the large-signal hysteresis displays a rather strong frequency dependence, ${ }^{11}$ both the large-signal hysteresis curve and the small signal response under bias have to be measured with the same frequency, i.e., the frequency used to trace out the hysteresis curve that is responsible for domain switching processes, $f_{\text {hys }}=f_{C-V}$. Therefore, the results obtained are only valid at that particular frequency.

Analogous observations could be made for ferroelectric bulk ceramics. In contrast to the thin films the electric fields were at least one order of magnitude smaller than for thin films. The coercive field for soft PZT ceramics varies between 8 and $14 \mathrm{kV} / \mathrm{cm}$ depending on the composition, i.e. the zirconium to titanium ratio. Ti-rich compositions usually exhibit higher coercive voltages. In ferroelectric thin films, however, the coercive fields are considerably higher. For PZT thin films, the coercive voltage is usually of the order of $100 \mathrm{kV} / \mathrm{cm}$. The application of an ac field of $50 \mathrm{mV}$ amplitude (as done during the measurement of a $C-V$ curve) to a film of typical thickness of $\sim 200 \mathrm{~nm}$ already constitutes the 
considerable electric field of $2.5 \mathrm{kV} / \mathrm{cm}$. This is contrasted with the field of $10^{-3} \mathrm{kV} / \mathrm{cm}$ that is applied to a $0.5 \mathrm{~mm}$ thick PZT ceramic during the measurement of its smallsignal capacitance. While for bulk ceramics the notion that the small superimposed ac field only generates small reversible oscillations of domain walls appears to be immediately self-evident, the factor of 3 orders of magnitude in the field strength in ferroelectric thin films calls for further justification.

Figure 1(b) displays the amplitude dependence of the relative permittivity of a PZT bulk ceramic sample (thickness $250 \mu \mathrm{m})$. Qualitatively, the overall appearance of the curves was identical to the one measured on ferroelectric thin films [Fig. 1(a)]. The same tendency for the peak positions to shift for increasing ac levels of the small signal amplitude as in Fig. 1(a) was observed. The maxima increased for increasing ac amplitude while at the same time their position shifted towards the $E=0$ axis. The bridge used for this measurement, however, only allowed a maximal ac amplitude of 10 V. Nevertheless, the shift of the maximum towards the $E$ $=0$ axis was already clearly discernible. For very high ac amplitudes levels, i.e., strong fields, one should therefore measure a small-signal capacitance characteristic where the two distinct peaks that were observed for low ac amplitudes had merged together to form a single peak.

Assuming the relation $E=U / d$ to be valid, the field inside a ferroelectric thin film should be several orders of magnitude higher than in bulk ceramics during the measurement of the small-signal capacitance. This assumption taken together with the observation of the amplitude dependence of the small-signal capacitance in bulk ceramics implies that a small-signal capacitance measurement on ferroelectric thin films should look radically different than a measurement on bulk. One would expect that such a measurement would only display a single peak centered at $E=0$ and not the characteristic double peak observed in $C-V$ measurements. The question therefore is: Why does one observe the characteristics of a $C-V$ curve also in thin films?

Following the logic of the last paragraph, one would expect the $C-V$ curves measured in ferroelectric thin films to display the features observed in bulk ceramics for high ac levels already at low ac levels because the same trend for increasing ac levels is also observed in PZT bulk ceramics and the electric fields inside a thin films should be much larger than in bulk ceramics. For low ac levels, the observed $C-V$ curve is not in accordance with this logic. It displays the completely analogous features of a $C-V$ curve measured on a bulk sample. Therefore, the measured amplitude dependence in thin films can be taken as evidence that the electric field acting on the ferroelectric bulk of the film is actually much smaller than expected from the relation $E=U / d$ or that the threshold fields for domain wall nucleation and movement are considerably higher in thin films than in bulk ceramics.

The existence of a surface interface layer has been proposed to explain anomalies in the behavior of ferroelectric bulk materials. Several models have been proposed to explain the nature of this interface layer. The interface layer is either believed to be a space charge layer, ${ }^{12}$ a Schottky ex- haustion barrier, ${ }^{13}$ or a chemically or mechanically distorted layer which does not participate in the polarization reversal process but gives rise to interface charges. ${ }^{14}$ Recently, several authors assumed the existence of a nonferroelectric interface layer to explain the discrepancies in electrical properties between thin films and bulk ceramics, like the thickness dependence of the coercive field or the switching properties of ferroelectric thin films. ${ }^{15-17}$ The decrease of the dielectric constant $\varepsilon$ with decreasing film thickness of $\mathrm{SrTiO}_{3}$ films is also believed to be due to the existence of an interface layer, ${ }^{18}$ as is the observation that the dielectric constant is much smaller in thin films compared to $\mathrm{SrTiO}_{3}$ single crystals. ${ }^{19}$ Several models have been proposed to account for the existence of such an interface layer, like nonstoichiometric growth of the ferroelectric at the interface between the electrodes and the film due to interdiffusion, a stress-induced interface layer ${ }^{20}$ or sputter damage by incorporation of argon ions into the surface layer of the ferroelectric film during the top electrode sputtering process. ${ }^{21}$ Even though it has not yet been achieved to provide direct verification of a dead interface layer, there has been ample indirect evidence for its existence. Therefore, it appears reasonable to assume that a possibly large fraction of the applied voltage already drops over this interface layer and that the ferroelectric bulk of the film is actually exposed to smaller electric fields. However, a simple dielectric interface layer alone cannot account for the observed thickness dependence of the coercive field in ferroelectric thin films since such a layer would only result in a tilt of the ferroelectric hysteresis loop. ${ }^{15,16}$ Other factors also appear to hinder the domain wall motion in ferroelectric thin films. In the literature, several mechanisms are discussed: Nearby electrode nucleation, based on the idea that the film thickness limits the size of the critical nuclei for domain reversal, ${ }^{22}$ thermoactivated domain wall motion, where the domain wall velocity undergoes a crossover between the Miller-Weinreich form ${ }^{23}$ to a new direct exponential field dependence when the film thickness $L$ becomes smaller than the critical nucleus size $r^{*},{ }^{16}$ surface pinning size effect, ${ }^{24}$ where the domain wall motion is hindered by its interaction with surface defects and the depletion assisted nucleation model. $^{25}$

All experimental and theoretical evidence seems to suggest that besides a reduction of the internal bulk field, due to the existence of a thin dielectric layer of low permittivity, size effects also hinder the domain wall motion so that the interpretation of the small-signal capacitance as a measure for reversible polarization processes appears to be justified for thin films.

\section{B. Recoil measurements and $C-V$ curves}

A direct method to measure reversible and irreversible contributions is the measurement of recoil curves, as illustrated in Fig. 3(a). The corresponding voltage applied to the sample is shown in Fig. 3(b). During the first period, the state of negative remanent polarization was established. Then the voltage was increased to $V$ and when the point $\left(P_{\text {tot }}, V\right)$ was reached, the voltage was returned to zero. By integrating the current, the polarization was calculated. The polarization 

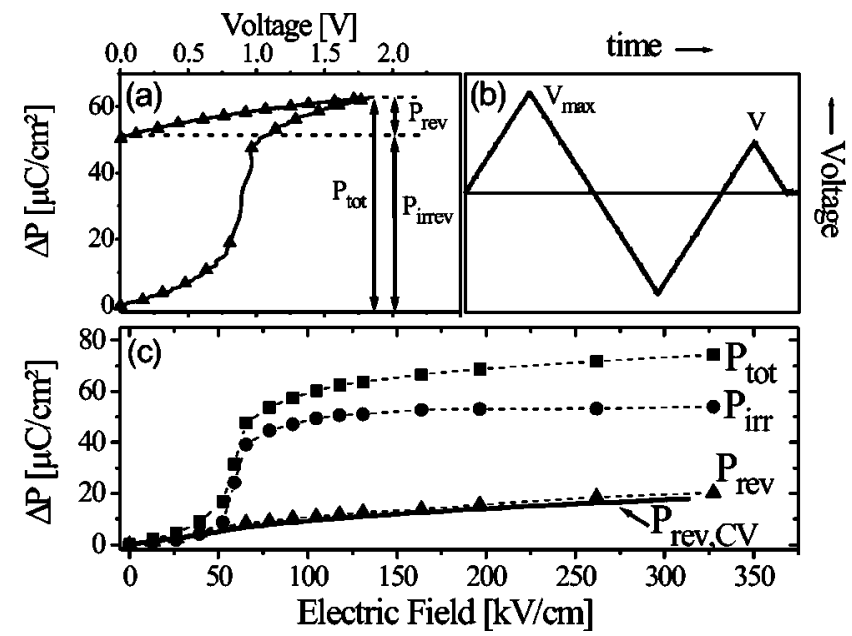

FIG. 3. (a) Example of recoil loops measured on a PZT 20/80 thin film with $f=1 \mathrm{~Hz}$. The polarization components, $P_{\text {rev }}$ and $P_{\text {irrev }}$, are also indicated in the graph; (b) the waveform used to separate reversible and irreversible polarization components; (c) reversible, irreversible and total polarization changes as function of maximal applied electric field for a PZT 20/80 thin film. The polarization changes have been measured at $1 \mathrm{~Hz}$. Also shown is the reversible polarization as obtained from integrating a $C-V$ curve.

in Fig. 3(a) is displayed relative to the state of negative remanent polarization. In this way, the irreversible polarization, $P_{\text {irrev }}$, is given by the intercept of the recoil curve with the axis of ordinates. The reversible polarization, $P_{\text {rev }}$, is calculated as the difference between $P_{\text {tot }}$ and $P_{\text {irrev }}$. The result of such a measurement for various voltages $V$ is shown in Fig. 3(c) for a PZT 20/80 thin film. A frequency of $1 \mathrm{~Hz}$ was used to measure the polarization. For other compositions the results were identical.

For fields higher than the coercive field of the film ( $\approx 65 \mathrm{kV} / \mathrm{cm}$ ), the increase in the total polarization is entirely due to reversible polarization changes, thus $P_{\text {irrev }}$ is parallel to the $x$ axis. In this regime, most domain walls were driven out of the material and only reversible dielectric processes contributed to the polarization. Only $P_{\text {irrev }}$ can be effectively used to store information in a ferroelectric random access memory (FeRAM).

As detailed in Sec. I, the integrated $C-V$ curve should be a measure for reversible polarization contributions. Figure 3(c) also shows the reversible polarization (black solid curve) obtained by integrating a $C-V$ curve measured at $f$ $=1 \mathrm{~Hz}\left(f_{\mathrm{ac}}=10 \mathrm{kHz}, U_{\mathrm{ac}}=100 \mathrm{mV}\right)$. The lower branch of the hysteretic integrated $C-V$ curve was chosen since by construction (i.e., integration), the reversible polarization $P_{C-V \text {,rev }}$ only conveys information about polarization differences. The reversible polarization obtained from $C-V$ measurements was in very good agreement with the reversible polarization $P_{\text {rev }}$ obtained directly from recoil measurements, thus giving strong support for the proposed interpretation of capacitance-voltage measurements. The small difference observed could be attributed to the fact that the current measured in recoil experiments also contained higher harmonics of the exciting voltage sequence while the capacitance measurement only measured the first harmonic.

For bulk ceramics, a very similar behavior was observed. Figure 4 displays results obtained from recoil measurements

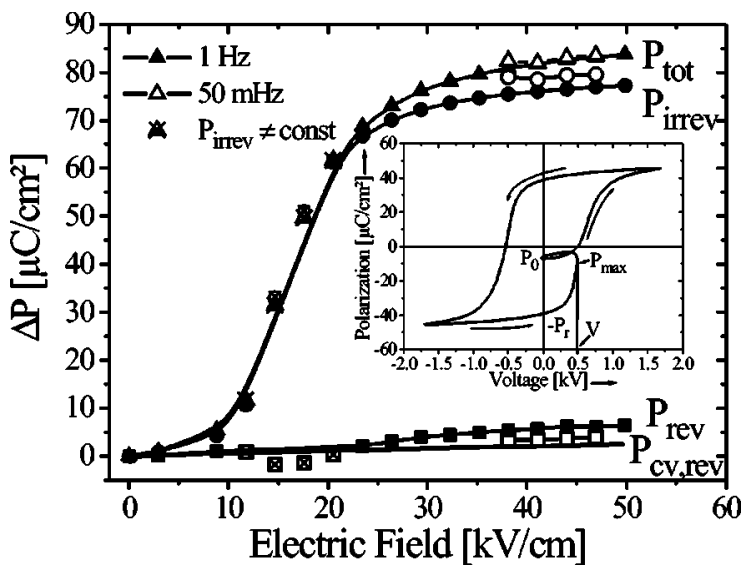

FIG. 4. Reversible, irreversible, and total polarization changes as function of maximal applied electric field for a $\mathrm{Nd}$ doped rhombohedral $(\mathrm{Zr}=0.58)$ PZT ceramic. Also shown is the reversible polarization as obtained from integrating a $C-V$ curve. The recoil measurements have been performed at $f=1 \mathrm{~Hz}$. Some results for $f=50 \mathrm{mHz}$ are also shown. The inset displays a recoil curve for $V=500 \mathrm{~V}\left(V_{\max }=1700 \mathrm{~V}, f=1 \mathrm{~Hz}\right)$.

on a Nd-doped PZT ceramic of rhombohedral composition $(\mathrm{Zr}=0.58)$. The polarization was measured at $1 \mathrm{~Hz}$. Again, for fields higher than the coercive field, the irreversible polarization was approximately constant. This method, however, is not reliable in the vicinity of the coercive field. The reason is apparent from the inset in Fig. 4 where a recoil curve for $V=500 \mathrm{~V}$ is shown $\left(V_{\max }=1700 \mathrm{~V}\right)$. At the maximal applied voltage $V$ of the recoil curve, the value of the polarization amounted to $P_{\max }$. As the field was returned to zero, however, the polarization continued to increase. As a consequence the polarization $P_{0}$ at zero field was actually higher than $P_{\text {max }}$, thus the reversible polarization $P_{\text {rev }}$ $=P_{\max }-P_{0}$ became negative. At fields close to $E_{c}$, the domain configuration was very unstable since the material was on the verge of switching to the opposite polarization state. Thus, though the external field was decreasing again, the material continued to switch into the opposite polarization state, entailing the increase in polarization. The prime assumption that no irreversible processes occurred along the recoil curve (in other words $P_{\text {irrev }}=$ const), was not valid and the reversible polarization could not reliably be determined in this region.

Also shown in the Fig. 4 is the reversible polarization obtained from an integrated $C-V$ curve. The $C-V$ dependence, however, was recorded at $\approx 1 \mathrm{mHz}$. The lock-in technique was not available for bulk materials (due to the higher voltage levels involved) so that only the slower bridge method could be used. The reversible polarization obtained from recoil measurements deviated substantially from the integrated $C-V$ curve. This, however, was entirely due to the different frequencies used to measure the data points, as seen from the recoil measurement data points measured at 50 $\mathrm{mHz}$ (open symbols), which are much closer to $P_{C-V \text {,rev }}$. It can therefore be safely inferred that the integrated $C-V$ curve is a good measure for reversible polarization contributions in bulk ceramics as well (when the frequency dependence of the large and small-signal measurements is properly taken into account). 


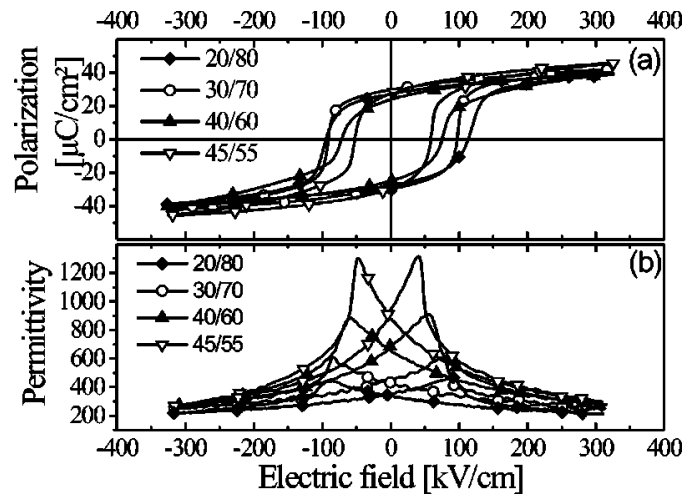

FIG. 5. (a) Hysteresis curves of PZT films of different composition; (b) relative permittivity as function of field for the same films $\left(f_{\mathrm{ac}}=10 \mathrm{kHz}\right)$. The frequency of the (triangular) large-signal was $1 \mathrm{~Hz}$ for all measurements.

\section{Composition influence}

\section{Thin films}

A series of PZT thin films of different Zr:Ti ratio was prepared to investigate the influence of the composition on reversible polarization contributions. The Ti content was varied between $45 \%$ and $80 \%$. Figure 5(a) shows the hysteresis curves measured on these samples (measured at $1 \mathrm{~Hz}$ ). The highest remanent polarization $\left(29.8 \mu \mathrm{C} / \mathrm{cm}^{2}\right)$ was determined for the $30 / 70$ film, while the lowest $P_{r}$ of 23.9 $\mu \mathrm{C} / \mathrm{cm}^{2}$ was measured for the $40 / 60 \mathrm{film}$. The remanent polarization of the $45 / 55 \mathrm{film}$ amounted to $27.6 \mu \mathrm{C} / \mathrm{cm}^{2} . P_{r}$ of the 20/80 film, $27.4 \mu \mathrm{C} / \mathrm{cm}^{2}$, was slightly lower than that of the 30/70 composition. The coercive field was highest for the $20 / 80$ film $\left(E_{c}=104.6 \mathrm{kV} / \mathrm{cm}\right)$ and decreased with decreasing $\mathrm{Ti}$ content to $55.5 \mathrm{kV} / \mathrm{cm}$ for the $45 / 55$ composition. Similar values for these compositions were reported..$^{26,27}$ The remanent polarization for the more tetragonal compositions, however, was reported to be smaller than that of near morphotropic compositions ${ }^{26}$ while for epitaxial PZT films on $\mathrm{SrRuO}_{3} / \mathrm{SrTiO}_{3}(001)$ substrates $P_{r}$ increased with increasing Ti content. ${ }^{27}$

Figure 5(b) displays the relative permittivity as a function of the applied electric field. The $\varepsilon-V$ curves were measured with the lock-in amplifier technique at $1 \mathrm{~Hz}\left(f_{\mathrm{ac}}=10\right.$ $\mathrm{kHz}$ ). The relative permittivity increased with decreasing Ti content in accordance with other reports. ${ }^{26,27}$

From the $C-V$ curves, the reversible polarization was obtained by use of Eq. (1) and is shown in Fig. 6 for the different PZT compositions investigated. At the point of positive saturation the reversible polarization increased from $2.4 \mu \mathrm{C} / \mathrm{cm}^{2}$ over $12.5 \mu \mathrm{C} / \mathrm{cm}^{2}$ to $14.8 \mu \mathrm{C} / \mathrm{cm}^{2}$ for the $30 /$ $70,40 / 60$, and 45/55 composition, respectively. The change of polarization of the hysteresis (i.e., the sum of reversible and irreversible contributions) in this composition range, however, is much smaller [Fig. 5(a)]. It can therefore be concluded that the reversible polarization contributions increase with decreasing Ti content [to be more precise, $P_{\text {rev }}$ increases as the morphotropic phase boundary (MPB) is approached].

It is interesting to note that the extrinsic contributions in PZT films also increase as the MPB is approached. Hiboux

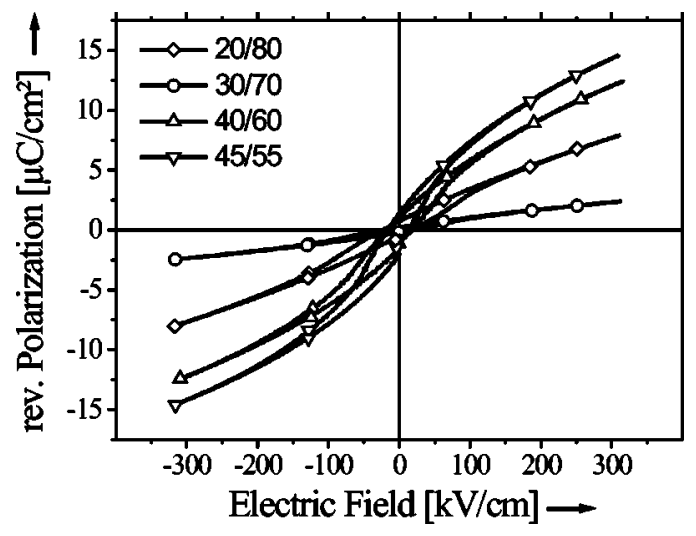

FIG. 6. Reversible polarization contributions for different PZT film compositions.

et al. separated the domain wall from the intrinsic contributions to the permittivity by assuming that the permittivity at saturation is equal to the lattice contribution based on the notion that at this point all domain walls are driven out of the material. ${ }^{26}$ The permittivity measured in excess of this value is then equal to the extrinsic contribution. It was observed that the domain wall contribution increases with decreasing Ti content and reaches a maximum at the 45/55 composition (for higher $\mathrm{Zr}$ contents it decreases again). The data presented above are in complete agreement with their findings. For PZT compositions near the morphotropic phase boundary the domain wall density and/or mobility is thus significantly enhanced.

\section{Bulk ceramics}

Analogous experiments were performed on $\mathrm{PbZr}_{x} \mathrm{Ti}_{1-x} \mathrm{O}_{3}$ bulk ceramics with compositions around the morphotropic phase boundary. Figure 7(a) displays the relative permittivity as a function of dc bias for a tetragonal ( $x$ $=0.48)$, a morphotropic $(x=0.52)$, and a rhombohedral $(x$ $=0.58$ ) sample. The highest permittivity was reached for the sample of composition close to the morphotropic phase boundary with $\varepsilon_{r}=1013$. For the tetragonal and rhombohedral sample $\varepsilon_{r}$ was determined to 783 and 711 , respectively (at $E=0$ ). The additional "humps" observed in the $\varepsilon-E$ curves were also observed by others in ferroelectric ceramics

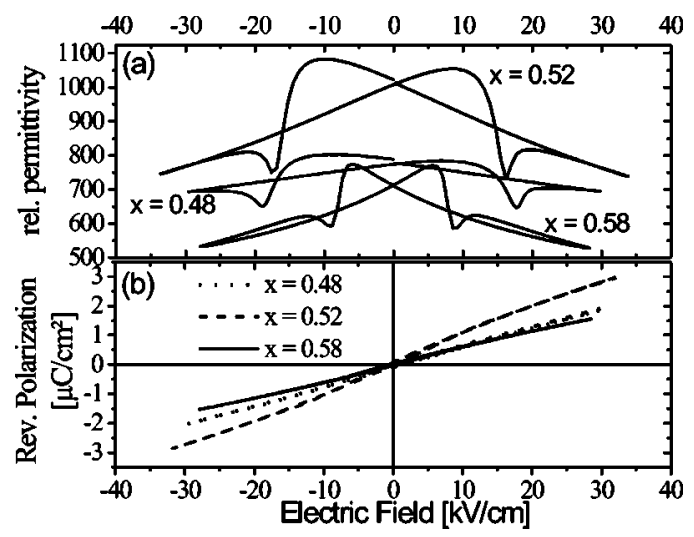

FIG. 7. (a) Relative permittivity and (b) reversible polarization of $\mathrm{PbZr}_{x} \mathrm{Ti}_{1-x} \mathrm{O}_{3}$ (2\% Nd doped) samples. 


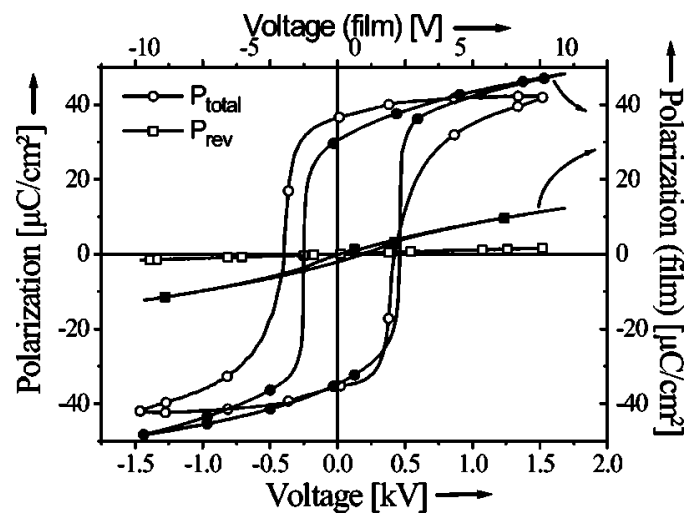

FIG. 8. Comparison of total polarization (circle) to reversible polarization (square) for a PZT $(x=0.56)$ ceramic (open symbols) and a thin film (Zr:Ti $=45: 55)$ (closed symbols).

and explained by different coercive fields for $180^{\circ}$ and non$180^{\circ}$ domains. $^{28}$ Their absence in ferroelectric thin films could be taken as evidence for suppressed non- $180^{\circ}$ domain switching in thin films. ${ }^{29}$

Figure 7(b) shows the reversible polarization contributions determined by integration of the capacitance-voltage curves of Fig. 7(a). The result is very similar to the one observed for the thin film samples. The highest reversible contributions were observed for the morphotropic composition, while the tetragonal and rhombohedral samples exhibited smaller reversible polarization contributions. From this perspective, thin films and bulk ceramics show the same qualitative behavior.

\section{Comparison bulk thin film}

However, a difference was observed when the magnitude of the reversible polarization was related to the total polarization. Figure 8 shows a comparison of the reversible polarization to the total polarization for bulk ceramics (open symbols) and thin films (closed symbols). It can clearly be seen that while in bulk ceramics the reversible contribution to the total polarization is almost negligible, it amounts to almost one third in thin film samples.

TEM studies of PZT 20/80 thin films and bulk ceramics showed that the $90^{\circ}$ type domain morphology is to first order similar for both systems. However, the mobility of $90^{\circ}$ domain walls is greatly reduced in thin films. ${ }^{30}$ Atomic force microscopy (AFM) studies on epitaxial PZT films showed that $90^{\circ}$ domain walls form a complicated network that inhibits the motion of single walls. ${ }^{31,32}$ In view of the presented results, it is therefore suggested that a large part of the ferroelectric response of thin films is due to reversible domain wall motion, i.e., bending of otherwise immobile domain walls while in bulk ceramics the walls are easily moved by the application of an external field.

\section{E. Reversible contributions during fatigue}

Figure 9(a) shows a typical example of a fatigue measurement performed on a PZT 30/70 film with Pt electrodes. The fatigue treatment was performed with rectangular pulses of amplitude $6.5 \mathrm{~V}$ and frequency $1 \mathrm{kHz}$. The hysteresis curves used to determine the decrease of the remanent polar-

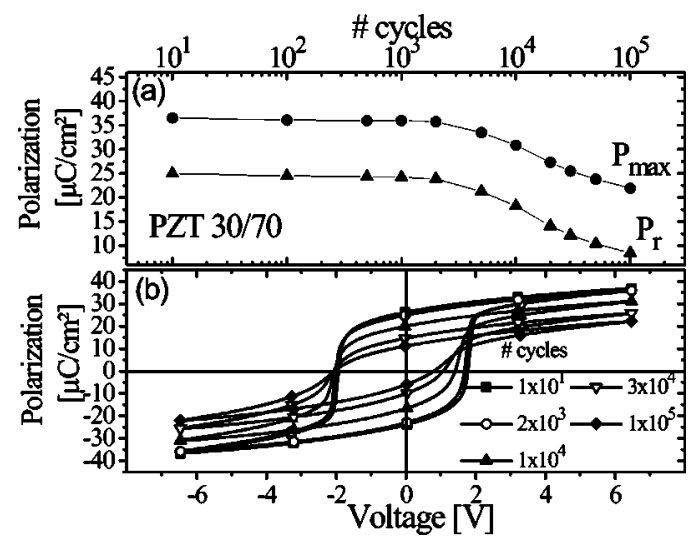

FIG. 9. (a) Fatigue measurement of a 30/70 PZT film (b) Corresponding hysteresis curves.

ization were measured at $1 \mathrm{~Hz}$ (also with $6.5 \mathrm{~V}$ amplitude). After only 10000 polarization switches the material exhibited a decline of the remanent polarization which was accompanied by an increased tilt of the hysteresis loops [Fig. 9(b)]. Additionally, a shift into negative voltage direction was also observed. For this kind of material, the onset of fatigue was reported between $10^{3}$ and $10^{6}$ cycles. $^{33}$

In addition to the hysteresis curves, capacitance voltage curves were also measured using the lock-in technique $(f$ $=1 \mathrm{~Hz}, f_{\mathrm{ac}}=10 \mathrm{kHz}, U_{\mathrm{ac}}=100 \mathrm{mV}$ ) which are displayed in Fig. 10. (It is very difficult to measure $C-V$ curves during a fatigue treatment with the bridge method due to the high dc stress imposed which often leads to a dielectric breakdown and subsequent shorting of the capacitor structure under investigation.) Clearly, the permittivity of the film decreased with the number of cycles. The same behavior was also observed for other PZT compositions and has also been found by others. ${ }^{34}$

Surprisingly, the integrated response, i.e., the reversible polarization contributions, remained practically unchanged during the fatigue treatment. Thus, the decrease of remanent polarization observed during a fatigue treatment was completely due to a decrease of the irreversible polarization contributions. The data available, however, do not present any clue as to what might be the underlying microscopic mechanism. The wall pinning mechanism, where the domain walls

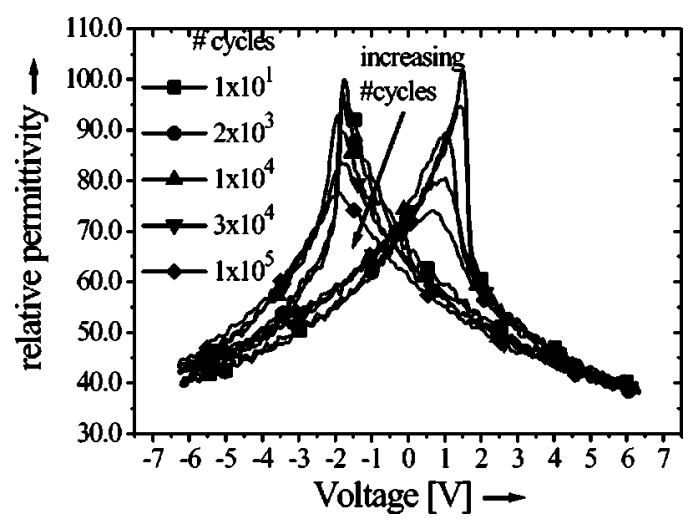

FIG. 10. Evolution of the small-signal dielectric response of a PZT 30/70 thin films during a fatigue treatment. 
are immobilized during the fatigue treatment presumably by free carriers that are subsequently trapped, ${ }^{35}$ predicts an increase of the permittivity in the saturation regime since during a fatigue treatment more and more domain walls are pinned that cease to contribute to the polarization switching but continue to contribute to the permittivity by wall bending. A careful inspection of the data presented in Fig. 10 indeed showed an increase of the permittivity at $-E_{\max }$. For $+E_{\max }$, however, no such increase was observed. The "seed inhibition" mechanism, ${ }^{34}$ on the other hand, where the switching of a domain is suppressed by inhibiting the formation or growth of new oppositely oriented seeds, predicts that the fatigued state should consist of relatively large singledomain regions. A pattern of this type has recently been reported. ${ }^{36}$ Consequently, the domain wall density should decrease during a fatigue treatment, and thus their contribution to the permittivity. The described observations are in more agreement with the latter theory.

\section{CONCLUSIONS}

In this article, it was demonstrated by experimental evidence that the integrated capacitance-voltage curve can be interpreted as a measure for reversible polarization processes (when proper care is taken of the frequency dependence of the measured quantities). This interpretation is valid in bulk ceramics as well as in thin films.

The method was used to investigate the composition dependence as well as the effect of fatigue on the reversible polarization. The composition dependence is very similar for bulk ceramics and thin films. During fatigue, the reversible polarization contributions remain practically constant, thus the reduction of the total polarization is almost entirely due to a decrease of the irreversible polarization component. The investigation revealed a much higher reversible contribution in thin films compared to bulk ceramics which can be taken as evidence that the domain wall motion in thin films is limited compared to bulk ceramics and only reversible domain wall motions prevail.

\section{ACKNOWLEDGMENTS}

The authors would like to thank Dr. C. Heilig and Professor Dr. K. H. Härdtl of the Institut für Werkstoffe der Elektrotechnik, University of Karlsruhe for providing the PZT bulk ceramics samples.
${ }^{1}$ D. Damjanovic, J. Appl. Phys. 82, 1788 (1997).

${ }^{2}$ D. Taylor and D. Damjanovic, J. Appl. Phys. 82, 1973 (1997).

${ }^{3}$ O. Boser and D. Beshers, Mater. Res. Soc. Symp. Proc. 82, 441 (1987).

${ }^{4}$ O. Boser, J. Appl. Phys. 62, 1344 (1987).

${ }^{5}$ D. Bolten, U. Böttger, T. Schneller, M. Grossmann, O. Lohse, and R. Waser, Appl. Phys. Lett. 77, 3830 (2000).

${ }^{6}$ S. Majumder, B. Roy, R. Katiyar, and S. Krupanidhi, Appl. Phys. Lett. 79, 239 (2001).

${ }^{7}$ D. Crew, P. McCormick, and R. Street, J. Appl. Phys. 86, 3278 (1999).

${ }^{8}$ R. Bozorth, Ferromagnetism, 3rd ed. (Van Nostrand, New York, 1955).

${ }^{9}$ R. Cammarano, P. McCormick, and R. Street, J. Phys. D 29, 2327 (1996).

${ }^{10}$ C. Heilig and K. Härdtl, Proc. IEEE Symp. Appl. Ferroelectrics 596, 504 (1998).

${ }^{11}$ D. Bolten, O. Lohse, M. Grossmann, and R. Waser, Ferroelectrics 221, 251 (1999).

${ }^{12}$ W. Känzig, Phys. Rev. A 98, 549 (1955).

${ }^{13}$ S. Triebwasser, Phys. Rev. A 118, 100 (1960).

${ }^{14}$ M. Drougard and R. Landauer, J. Appl. Phys. 30, 1663 (1959).

${ }^{15}$ J. Cillessen, M. Prins, and R. Wolf, J. Appl. Phys. 81, 2777 (1997).

${ }^{16}$ A. Tagantsev, Integr. Ferroelectr. 16, 237 (1997).

${ }^{17}$ A. Bratkovsky and A. Levanyuk, Phys. Rev. Lett. 84, 3177 (2000).

${ }^{18}$ K. Abe and S. Komatsu, Jpn. J. Appl. Phys., Part 1 32, 4186 (1993).

${ }^{19}$ C. Zhou and D. Newns, J. Appl. Phys. 82, 3081 (1997).

${ }^{20}$ S. Desu, J. Electrochem. Soc. 140, 2981 (1993).

${ }^{21}$ S.-K. Hong, H. G. Yang, and H. J. Kim, J. Korean Phys. Soc. 32, S1525 (1998).

${ }^{22}$ H. Kay and J. Dunn, Philos. Mag. 7, 2027 (1962).

${ }^{23}$ R. Miller and G. Weinreich, Phys. Rev. 117, 1460 (1960).

${ }^{24}$ N. Lebedev and A. Sigov, Integr. Ferroelectr. 4, 21 (1994).

${ }^{25}$ A. Tagantsev, C. Pawlaczyk, K. Brooks, and N. Setter, Integr. Ferroelectr. 4, 1 (1994).

${ }^{26}$ S. Hiboux, P. Muralt, and T. Maeder, J. Mater. Res. 14, 4307 (1999).

${ }^{27}$ C. Foster, G.-R. Bai, R. Csencsitis, J. Vetrone, R. Jammy, L. Wills, E. Carr, and J. Amano, J. Appl. Phys. 81, 2349 (1997).

${ }^{28}$ N. Bar-Chaim, M. Brunstein, J. Grünberg, and A. Seidman, J. Appl. Phys. 45, 2398 (1974).

${ }^{29}$ D. Damjanovic, Rep. Prog. Phys. 61, 1267 (1998).

${ }^{30}$ B. Tuttle, T. Headley, C. Drewien, J. Michael, J. Voigt, and T. Garino, Ferroelectrics 221, 209 (1999).

${ }^{31}$ K. Lee, J. Choi, J. Lee, and S. Baik, J. Appl. Phys. 90, 4095 (2001).

${ }^{32}$ C. Ganpule, V. Nagarajan, B. Hill, A. Roytuburd, E. Williams, R. Ramesh, S. Alpay, A. Roelofs, R. Waser, and L. M. Eng, J. Appl. Phys. 91, 1477 (2002).

${ }^{33}$ A. Tagantsev, I. Stolichnov, E. Colla, and N. Setter, J. Appl. Phys. 90, 1387 (2001)

${ }^{34}$ E. Colla, D. Taylor, A. Taganstev, and N. Setter, Appl. Phys. Lett. 72, 2478 (1998).

${ }^{35}$ W. Warren, D. Dimos, B. Tuttle, R. Nasby, and G. Pike, Appl. Phys. Lett. 65, 1018 (1994).

${ }^{36}$ E. Colla, S. Hong, D. Taylor, A. Taganstev, and N. Setter, Appl. Phys. Lett. 72, 2763 (1998). 